PROCEEDINGS OF THE

AMERICAN MATHEMATICAL SOCIETY

Volume 132, Number 5, Pages 1501-1506

S 0002-9939(03)07213-7

Article electronically published on September 18, 2003

\title{
INDUCED NORMS, STATES, AND NUMERICAL RANGES
}

\author{
CHI-KWONG LI, EDWARD POON, AND HANS SCHNEIDER
}

(Communicated by Joseph A. Ball)

\begin{abstract}
It is shown that two induced norms are the same if and only if the corresponding norm numerical ranges or radii are the same, which in turn is equivalent to the vector states and mixed states arising from the norms being the same. The proofs depend on an auxiliary result of independent interest which concerns when two closed convex sets in a topological vector space are multiples of each other.
\end{abstract}

\section{INTRODUCTION}

Let $\mathbb{F}$ be the complex field $\mathbb{C}$ or the real field $\mathbb{R}$, and let $\mathbf{X}$ be a vector space over $\mathbb{F}$ equipped with the norm $\nu$. The induced operator norm on the space $B(\mathbf{X})$ of bounded linear operators on $\mathbf{X}$ is defined as

$$
\|A\|=\sup \{\nu(A x): x \in \mathbf{X}, \nu(x) \leq 1\},
$$

the dual norm $\nu^{*}$ on the dual space $\mathbf{X}^{*}$ of $\mathbf{X}$ is defined by

$$
\nu^{*}(f)=\sup \{|f(x)|: x \in \mathbf{X}, \nu(x) \leq 1\},
$$

and the dual norm $\|\cdot\|^{*}$ on the dual space $B(\mathbf{X})^{*}$ is defined by

$$
\|F\|^{*}=\sup \{|F(A)|: A \in B(\mathbf{X}),\|A\| \leq 1\} .
$$

For each $(x, f) \in \mathbf{X} \times \mathbf{X}^{*}$, denote by $x \otimes f$ the rank one operator in $B(\mathbf{X})$ such that $(x \otimes f)(y)=f(y) x$ for any $y \in \mathbf{X}$. Then the set of vector states and the set of (mixed) states are, respectively,

$$
\mathcal{V}=\left\{x \otimes f \in \mathbf{X} \times \mathbf{X}^{*}: f(x)=\nu^{*}(f) \nu(x)=1\right\}
$$

and

$$
\mathcal{S}=\left\{F \in B(\mathbf{X})^{*}:\|F\|^{*}=F(I)=1\right\} .
$$

We shall identify $\mathcal{V}$ with a subset of $\mathcal{S}$ by defining $(x \otimes f)(A)=f(A x)$ for all $A \in B(\mathbf{X})$ and $x \otimes f \in \mathbf{X} \times \mathbf{X}^{*}$. Associated with $\nu$, via $\mathcal{V}$ and $\mathcal{S}$, are two norm numerical ranges of $A \in B(\mathbf{X})$ : the spatial numerical range

$$
W(A)=\{f(A x): x \otimes f \in \mathcal{V}\}
$$

and the algebraic numerical range

$$
V(A)=\{F(A): F \in \mathcal{S}\} .
$$

Received by the editors December 9, 2002 and, in revised form, January 22, 2003.

2000 Mathematics Subject Classification. Primary 47A12, 47A30, 15A60.

Key words and phrases. Induced norms, vector states, mixed states, norm numerical ranges. Research partially supported by an NSF grant. 
When $\mathbf{X}=\mathbb{C}^{n}$ and $\nu$ is the $\ell_{2}$ norm, $W(A)=V(A)$ is the classical numerical range of $A$; see [1], 2], [3], 4] for the background. It is known (see [2] Theorem 3, p. 83]) that $V(A)$ is the closure of the convex hull of $W(A)$, i.e.,

$$
V(A)=\overline{\operatorname{co}} W(A) .
$$

In view of this, both of these numerical ranges have the same associated norm numerical radius, namely,

$$
r(A)=\sup \{|\mu|: \mu \in W(A)\}=\sup \{|\mu|: \mu \in V(A)\},
$$

which (if $\mathbb{F}=\mathbb{C}$ ) is a norm on $B(\mathbf{X})$ such that

$$
e^{-1}\|A\| \leq r(A) \leq\|A\| \quad \text { for all } A \in B(\mathbf{X})
$$

see [2, p. 34].

Suppose $\left(\mathbf{X}, \nu_{1}\right)$ and $\left(\mathbf{X}, \nu_{2}\right)$ are normed spaces, where $\nu_{1}$ and $\nu_{2}$ are multiples of each other. It is easy to verify that they have the same induced norms on $B(\mathbf{X})$, and the numerical ranges (radii) and (vector) states are always the same. The purpose of this note is to show that the converse is also true. A key step in our proof is the following.

Theorem 1. Let $\nu_{1}$ and $\nu_{2}$ be two norms on $\mathbf{X}$. Let $\mathcal{V}_{i}$ be the set of vector states corresponding to the norm $\nu_{i}$ for $i=1,2$. Then $\nu_{1}$ and $\nu_{2}$ are multiples of each other if and only if for every nonzero $x \in \mathbf{X}$ there exists $f \in \mathbf{X}^{*}$ such that $x \otimes f \in \mathcal{V}_{1} \cap \mathcal{V}_{2}$.

In Theorem 1 we require only the existence of $f \in X^{*}$ (with respect to both $\nu_{1}^{*}$ and $\nu_{2}^{*}$ ) for any nonzero $x \in \mathbf{X}$ so that $x \otimes f \in \mathcal{V}_{1} \cap \mathcal{V}_{2}$ to conclude that $\nu_{1}$ and $\nu_{2}$ are multiples of each other. In other words, we do not assume a priori that the two norms $\nu_{1}$ and $\nu_{2}$ are equivalent. From Theorem 1 we can obtain the next theorem concerning two norms on $\mathbf{X}$ that give rise to the same numerical ranges (radii) or (vector) states in $B(\mathbf{X})$. In order to be able to compare the numerical ranges (radii) and (vector) states with respect to the two norms, we require $B(\mathbf{X})$ to be the same with respect to either of the two operator norms, i.e., $\|\cdot\|_{1}$ and $\|\cdot\|_{2}$ are equivalent. One readily checks that this condition holds if and only if $\nu_{1}$ and $\nu_{2}$ are equivalent $[\underline{6}$.

Theorem 2. Suppose $\nu_{1}$ and $\nu_{2}$ are equivalent norms on $\mathbf{X}$. Let $\mathcal{V}_{i}, \mathcal{S}_{j}, r_{j}, W_{j}$, and $V_{j}$ be the set of vector states, the set of states, the numerical radius, and the two types of numerical ranges corresponding to the norm $\nu_{i}$ for $i=1,2$. The following are equivalent:

(a) $\nu_{1}$ and $\nu_{2}$ are multiples of each other;

(b) $\mathcal{S}_{1}=\mathcal{S}_{2}$;

(c) $\mathcal{V}_{1}=\mathcal{V}_{2}$;

(d) $V_{1}(A)=V_{2}(A)$ for all $A \in B(\mathbf{X})$;

(e) $W_{1}(A)=W_{2}(A)$ for all $A \in B(\mathbf{X})$;

(f) $r_{1}(A)=r_{2}(A)$ for all $A \in B(\mathbf{X})$.

\section{Auxiliary Results and proofs of theorems}

Theorem 1 follows from the following more general result. 
Theorem 3. Let $\mathcal{K}_{1}$ and $\mathcal{K}_{2}$ be closed convex sets of a topological vector space $\mathbf{X}$ over $\mathbb{F}$. Suppose for each nonzero $x \in \mathbf{X}$ there exist $f_{x} \in \mathbf{X}^{*}, \mu_{1}(x), \mu_{2}(x)>0$ such that $\mu_{i}(x) x \in \mathcal{K}_{i}$ and

$$
f_{x}\left(\mu_{i}(x) x\right) \geq f_{x}(y) \quad \text { for all } y \in \mathcal{K}_{i}
$$

for $i=1,2$. Then $\mathcal{K}_{1}$ and $\mathcal{K}_{2}$ are multiples of each other.

Theorem 3 essentially says that if two closed convex sets in a topological vector space (TVS) share a common support functional in each direction, then the two sets are multiples of each other. Its proof depends on the following lemmas about convex sets in $\mathbb{R}^{2}$. The first lemma says that if two compact convex sets in $\mathbb{R}^{2}$ share a common support functional in each direction, then they actually have the same set of support functionals. The second lemma says that the boundary of a compact convex set in $\mathbb{R}^{2}$ is uniquely determined (up to a multiple) by the slopes of its support lines. It is not hard to see that these two lemmas combined give rise to the special case of Theorem 3 when the TVS is $\mathbb{R}^{2}$. It turns out that we can also deduce the general case from the two lemmas.

Lemma 4. Let $B_{1}$ and $B_{2}$ be two closed convex subsets of $\mathbb{R}^{2}$. For each nonzero $x \in \mathbb{R}^{2}$, and for $i=1,2$, define

$$
\begin{array}{r}
\mathcal{V}_{i}(x)=\left\{y \in \mathbb{R}^{2}: \exists \mu_{i}=\mu_{i}(x)>0 \text { such that } \mu_{i} x \in B_{i}\right. \\
\text { and } \left.y^{t} \mu_{i} x \geq y^{t} w \text { for all } w \in B_{i}\right\} .
\end{array}
$$

Suppose $\mathcal{V}_{1}(x) \cap \mathcal{V}_{2}(x) \neq \emptyset$ for all $x \neq 0$. Then $\mathcal{V}_{1}(x)=\mathcal{V}_{2}(x)$ for all $x \neq 0$.

Proof. The hypothesis

$$
\mathcal{V}_{1}(x) \cap \mathcal{V}_{2}(x) \neq \emptyset \quad \forall x \neq 0
$$

is equivalent to the existence of support lines to $B_{1}$ and $B_{2}$ at $\mu_{1} x$ and $\mu_{2} x$, respectively, with the same slope. (In particular, this implies that the origin lies in $B_{1} \cap B_{2}$.) Thus, the lemma's conclusion holds if and only if in each direction the two sets of support lines to $B_{1}$ and $B_{2}$ have the same slopes.

Fix an angle $\phi \in[0,2 \pi)$. By rotating both $B_{1}$ and $B_{2}$, we may suppose, without loss of generality, that $\phi=3 \pi / 2$. For all $\theta$ in a sufficiently small interval $I$ containing $3 \pi / 2$ we can define

$$
\begin{aligned}
& f_{-}(\theta)=\min \left\{m: y=m x+b \text { is a support line for } B_{1} \text { in the direction } \theta\right\}, \\
& f_{+}(\theta)=\max \left\{m: y=m x+b \text { is a support line for } B_{1} \text { in the direction } \theta\right\},
\end{aligned}
$$

and similarly we define $g_{-}(\theta), g_{+}(\theta)$ in terms of support lines for $B_{2}$. By the properties of convex functions [5, Theorem 24.1], both functions are increasing, $f_{-}$is left continuous, and $f_{+}$is right continuous. Moreover, if $\psi<\theta$, we have $f_{-}(\psi) \leq f_{+}(\psi) \leq f_{-}(\theta)$.

For directions $\theta \in I$, the condition (11) is equivalent to

$$
\left[f_{-}(\theta), f_{+}(\theta)\right] \cap\left[g_{-}(\theta), g_{+}(\theta)\right] \neq \emptyset \quad \forall \theta \in I .
$$

Fix $\theta \in I$. We claim $g_{-}(\theta) \geq f_{-}(\theta)$. Suppose not. Then $g_{-}(\theta)<f_{-}(\theta)$. So by the left continuity of $f_{-}$, there is some $\psi<\theta$ such that $g_{-}(\theta)<f_{-}(\psi) \leq f_{-}(\theta)$. Thus $g_{+}(\psi) \leq g_{-}(\theta)<f_{-}(\psi)$, contradicting (2). A similar argument shows that $g_{+}(\theta) \leq f_{+}(\theta)$. So we have

$$
\left[g_{-}(\theta), g_{+}(\theta)\right] \subset\left[f_{-}(\theta), f_{+}(\theta)\right] \quad \forall \theta \in I .
$$


Reversing the roles of $f$ and $g$ gives the reverse inclusion. So $\left[f_{-}(\theta), f_{+}(\theta)\right]=$ $\left[g_{-}(\theta), g_{+}(\theta)\right]$ for all $\theta \in I$, and, in particular, $\left[f_{-}(\phi), f_{+}(\phi)\right]=\left[g_{-}(\phi), g_{+}(\phi)\right]$. Thus the support lines for $B_{1}$ and $B_{2}$ in the direction $\phi$ have the same slopes; since $\phi$ was arbitrary, we conclude that $\mathcal{V}_{1}(x)=\mathcal{V}_{2}(x)$ for all $x \neq 0$.

Lemma 5. Let $B \subset \mathbb{R}^{2}$ be a compact convex set containing the origin in its interior. Let $r=r(\theta)$ be the polar curve whose graph is the boundary $\partial B$. Then the function $\log r^{2}$ is left- and right-differentiable at every angle $\theta$, and its one-sided derivatives are uniquely determined by the slopes of the support lines of $B$.

Proof. Let $y=f(x)$ be the Cartesian curve whose graph is the lower portion of $\partial B$. Then $f$ is a convex function, and hence left- and right-differentiable everywhere. Writing $\left(x_{0}, y_{0}\right)$ for $(0, f(0))$ and $r_{0}$ for $r(0)$, where $\theta$ measures the angle counterclockwise from the negative $y$-axis, we have

$$
\begin{aligned}
\left(r^{2}\right)_{+}^{\prime}(0) & =\lim _{\theta \rightarrow 0+} \frac{r^{2}-r_{0}^{2}}{\theta} \\
& =\lim _{\theta \rightarrow 0+} \frac{r \sin \theta}{\theta}\left[\left(x+x_{0}\right)+\left(y+y_{0}\right) \frac{y-y_{0}}{x-x_{0}}\right] \\
& =r_{0}\left(2 y_{0} f_{+}^{\prime}(0)\right) \\
& =-2 r_{0}^{2} f_{+}^{\prime}(0)
\end{aligned}
$$

where we have used the continuity of $r(\theta)$ and the right-differentiability of $f(x)$ in the penultimate equality. The same argument shows that $\left(r^{2}\right)_{-}^{\prime}(0)=-2 r_{0}^{2} f_{-}^{\prime}(0)$.

Let $B_{\theta}$ be the set obtained by rotating $B$ by an angle $\theta$ clockwise about the origin. Let $m_{-}(\theta)$ (respectively $m_{+}(\theta)$ ) be the minimal (respectively, maximal) slope of any line of support to $B_{\theta}$ at the intersection $z_{\theta}$ of $B_{\theta}$ and the negative $y$-axis. (Both $m_{-}(\theta)$ and $m_{+}(\theta)$ are finite since $B_{\theta}$ cannot have a vertical line of support at $z_{\theta}$.) By considering the rotated set $B_{\theta}$, it follows that $\left(r^{2}\right)_{ \pm}^{\prime}(\theta)=-2 r^{2} m_{ \pm}(\theta)$, and, in particular, $\left(\log r^{2}\right)_{ \pm}^{\prime}(\theta)=-2 m_{ \pm}(\theta)$ for all angles $\theta$, as desired.

Proof of Theorem 3 . We may assume that $\mathbb{F}=\mathbb{R}$ since $\mathbf{X}$ may be viewed as a real linear space. We must show that $\mu_{1}(x) / \mu_{2}(x)$ is a constant, independent of $x \in \mathbf{X}$. By way of contradiction, suppose these ratios differ at two vectors $x$ and $y$ in $\mathbf{X}$. Let $\mathbf{M}$ be a two-dimensional real linear subspace of $\mathbf{X}$ containing $x$ and $y$, and let $B_{i}=\mathcal{K}_{i} \cap \mathbf{M}$. Clearly, $B_{1}$ and $B_{2}$ are closed and convex; moreover, if $z \in \mathbf{M}$, we have, by assumption, the existence of $f \in X^{*}, \mu_{1}, \mu_{2}>0$ such that $\mu_{i} z \in \mathcal{K}_{i}$ and $f\left(\mu_{i} z\right) \geq f(w)$ for all $w \in \mathcal{K}_{i}$. In particular, $\left.f\right|_{\mathbf{M}}\left(\mu_{i} z\right) \geq f(w)$ for all $w \in B_{i}$; since any linear functional on $\mathbf{M}$ is realized by the inner product with a vector in $\mathbf{M}, B_{1}$ and $B_{2}$ satisfy the hypotheses of Lemma 4 Thus, the slopes of the supporting lines for $B_{1}$ and $B_{2}$ are the same. But by Lemma 5 the slopes of the supporting lines determine a compact convex set up to a scalar multiple; so $B_{1}$ and $B_{2}$ are scalar multiples of one another, contradicting $\mu_{1}(x) / \mu_{2}(x) \neq \mu_{1}(y) / \mu_{2}(y)$.

Proof of Theorem 11. The implication $(\Rightarrow)$ is clear. For the converse, let $\mathcal{K}_{i}$ be the unit norm ball of $\nu_{i}$ for $i=1,2$. Let $x \in \mathbf{X}$ be nonzero. By assumption, there is some $f \in \mathbf{X}^{*}$ such that $x \otimes f \in \mathcal{V}_{1} \cap \mathcal{V}_{2}$, that is,

$$
f(x)=\nu_{i}^{*}(f) \nu_{i}(x)=1 \quad \text { for } i=1,2 .
$$


Setting $\mu_{i}(x)=1 / \nu_{i}(x)$ gives

$$
f\left(\mu_{i}(x) x\right)=\nu_{i}^{*}(f)=\sup _{y \in \mathcal{K}_{i}} f(y) \quad \text { for } i=1,2 .
$$

So Theorem 3 applies.

Proof of Theorem 圆 $(a) \Rightarrow(b)$ : Clear.

$(b) \Rightarrow(c)$ : Assume (b). Let $\mathcal{R}$ be the set of functionals in $B(\mathbf{X})^{*}$ of the form $x \otimes f$. It suffices to show that $\mathcal{V}_{j}=\mathcal{S}_{j} \cap \mathcal{R}$ for $j=1,2$. To avoid clutter, we suppress the subscript $j$.

That $\mathcal{V} \subseteq \mathcal{S} \cap \mathcal{R}$ is clear. For the reverse inclusion, suppose $x \otimes f \in \mathcal{S}$; so $\|x \otimes f\|^{*}=1=(x \otimes f)(I)=f(x)$. Given $\epsilon>0$, choose $y \in \mathbf{X}$ and $g \in \mathbf{X}^{*}$ such that $\nu(y)=1=\nu^{*}(g), f(y)>(1-\epsilon) \nu^{*}(f)$, and $g(x)=\nu(x)$. Then $\|y \otimes g\| \leq$ $\nu(y) \nu^{*}(g)=1$; so

$$
1=\|x \otimes f\|^{*}=\sup \{|f(A x)|:\|A\| \leq 1\} \geq|f(g(x) y)|>(1-\epsilon) \nu^{*}(f) \nu(x) .
$$

Since $\epsilon$ is arbitrary, we have $\nu^{*}(f) \nu(x) \leq 1$. On the other hand,

$$
1=|f(x)| \leq \nu^{*}(f) \nu(x) .
$$

Therefore, $f(x)=1=\nu^{*}(f) \nu(x)$; that is, $x \otimes f \in \mathcal{V}$ as desired.

$(c) \Rightarrow(a)$ : Immediate from Theorem 1 .

$(a) \Rightarrow(d),(e)$ : Clear.

$(d),(e) \Rightarrow(f)$ : Clear.

$(f) \Rightarrow(a)$ : We prove the contrapositive. Suppose that $\nu_{1}$ and $\nu_{2}$ are not multiples of each other. Then there is a two-dimensional subspace $\mathbf{M} \subset \mathbf{X}$ on which the restrictions of $\nu_{1}$ and $\nu_{2}$ are not multiples of each other. By Theorem 1 there exists $z \in \mathbf{M}$ such that

$$
\text { there is no } f \in \mathbf{M}^{*} \text { such that } z \otimes f \in \mathcal{U}_{1} \cap \mathcal{U}_{2} \text {, }
$$

where $\mathcal{U}_{i}$ is the set of vector states for $\mu_{i}=\left.\nu_{i}\right|_{\mathbf{M}}$. By rescaling the unit norm balls $B_{i}$ of $\nu_{i}$ (since this does not affect the vector states) we may suppose $z=(0,-1)$ and $\nu_{1}(z)=\nu_{2}(z)=1$. Using the definitions for $f_{ \pm}(\theta), g_{ \pm}(\theta)$ in the proof of Lemma 4 we note that (3) is equivalent to

$$
\left[f_{-}(3 \pi / 2), f_{+}(3 \pi / 2)\right] \cap\left[g_{-}(3 \pi / 2), g_{+}(3 \pi / 2)\right]=\emptyset .
$$

Without loss of generality, suppose

$$
f_{-}(3 \pi / 2)>g_{+}(3 \pi / 2) \text {. }
$$

Claim. There exists $x \in \partial B_{1}$ in the third quadrant and $y \in \partial B_{2}$ in the fourth quadrant such that

(i) $y$ lies on a support line to $B_{1}$ at $x$, and

(ii) a support line to $B_{2}$ at $y$ separates $x$ from $B_{2}$.

Via the usual correspondence between vectors and functionals on $\mathbb{R}^{2}$, let $f$ (respectively $g$ ) be the functional corresponding to the normal of the support line in (i) (respectively (ii)) whose inner product with $x$ (respectively $y$ ) is 1 . It follows that $A=x \otimes f \in \mathcal{U}_{1}$ and $y \otimes g \in \mathcal{U}_{2}$. By the Hahn-Banach Theorem, we can extend $f$ and $g$ to functionals on all of $\mathbf{X}$ such that $x \otimes f \in \mathcal{V}_{1}$ and $y \otimes g \in \mathcal{V}_{2}$. Since

$$
r_{1}(A) \leq\|A\|_{1} \leq \nu_{1}(x) \nu_{1}^{*}(f)=1
$$

and $r_{1}(A) \geq(x \otimes f)(A)=1$, we have $r_{1}(A)=1$. On the other hand,

$$
r_{2}(A) \geq(y \otimes g)(x \otimes f)=g(x) f(y)>1,
$$


where the final inequality follows from the claim. Hence the two numerical radii are different, as desired.

It remains to prove the claim; so we suppose it is false and derive a contradiction. Let $x=x(\theta)$ denote the unique intersection of the boundary of $B_{1}$ and the ray emanating from the origin in the direction $\theta$. Denote the intersection of the support line to $B_{1}$ at $x$ with minimal slope $f_{-}(\theta)$ and the boundary of $B_{2}$ by $y=y(\theta)$. (By (41) and the properties of convex curves, such an intersection exists for $\theta$ sufficiently close to $3 \pi / 2$.) Let $\phi$ denote the direction of $y$. Since the claim is supposed false, every support line to $B_{2}$ at $y$ must have slope greater than $f_{-}(\theta)$; in particular,

$$
g_{+}(\phi) \geq f_{-}(\theta) .
$$

Let $\theta$ approach $3 \pi / 2$ from below; so $\phi$ approaches $3 \pi / 2$ from above. The left continuity of $f_{-}$and the right continuity of $g_{+}$imply $g_{+}(3 \pi / 2) \geq f_{-}(3 \pi / 2)$, con-

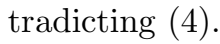

\section{ACKNOWLEDGMENT}

The first author thanks Professor David Lutzer for some helpful discussion concerning the formulation of Theorem 3 .

\section{REFERENCES}

[1] F. L. Bauer, On the field of values subordinate to a norm, Numer. Math. 4 (1962), 103-113. MR 26:2860

[2] F. F. Bonsall and J. Duncan, Numerical ranges of operators on normed spaces and of elements of normed algebras, London Mathematical Society Lecture Note Series 2, Cambridge University Press, Cambridge, 1971. MR 44:5779

[3] R. A. Horn and C. R. Johnson, Topics in Matrix Analysis, Cambridge University Press, Cambridge, 1991. MR 92e:15003

[4] N. Nirschl and H. Schneider, The Bauer fields of values of a matrix, Numer. Math. 6 (1964), 355-365. MR 31:871

[5] R. T. Rockafellar, Convex Analysis, Princeton University Press, Princeton, New Jersey, 1970. MR 43:445

[6] H. Schneider and W. G. Strang, Comparison theorems for supremum norms, Numer. Math. 4 (1962), 15-20. MR 24:A1917.

Department of Mathematics, College of William and Mary, P.O. Box 8795, WilliamsBURG, VIRGINIA 23187-8795

E-mail address: ckli@math.wm.edu

Department of Mathematics, Embry-Riddle Aeronautical University, 3200 Willow Creek Road, Prescott, Arizona 86301

E-mail address: edward.poon@erau.edu

Department of Mathematics, University of Wisconsin, Madison, Wisconsin 53706

E-mail address: hans@math.wisc.edu 